\title{
Testing Euclidean Minimum Spanning Trees in the Plane $* \dagger$
}

\author{
Artur Czumaj \\ Department of Computer Science \\ University of Warwick \\ Coventry CV4 7AL, United Kingdom \\ czumaj@dcs.warwick.ac.uk
}

\author{
Christian Sohler \\ Heinz Nixdorf Institute \\ Computer Science Department \\ University of Paderborn \\ D-33102 Paderborn, Germany \\ csohler@upb.de
}

\author{
Martin Ziegler \\ Heinz Nixdorf Institute \\ Computer Science Department \\ University of Paderborn \\ D-33102 Paderborn, Germany \\ ziegler@uni-paderborn.de
}

\begin{abstract}
Given a Euclidean graph $G$ over a set $P$ of $n$ points in the plane, we are interested in verifying whether $\mathrm{G}$ is a Euclidean minimum spanning tree (EMST) of $\mathrm{P}$ or $\mathrm{G}$ differs from it in more than $€ n$ edges. We assume that $G$ is given in adjacency list representation and the point/vertex set $P$ is given in an array. We present a property testing algorithm that accepts graph $G$ if it is an EMST of $P$ and that rejects with probability at least $\frac{2}{3}$ if $\mathrm{G}$ differs from every EMST of $P$ in more than $\epsilon n$ edges. Our algorithm runs in $\mathcal{O}\left(\sqrt{n / \epsilon} \cdot \log ^{2}(n / \epsilon)\right)$ time and has a query complexity of $\mathcal{O}(\sqrt{n / \epsilon} \cdot \log (n / \epsilon))$.
\end{abstract}

Keywords: Euclidean minimum spanning tree, property testing, randomized algorithms.

\footnotetext{
${ }^{*}$ The results presented in this paper appeared in a preliminary form as a part of the paper "Property testing in computational geometry," in Proceedings of the 8th Annual European Symposium on Algorithms (ESA'00), pages 155-166, volume 1879 of Lecture Notes in Computer Science, Springer-Verlag, Berlin, 2000 [6] and in the Ph.D. thesis of the second author "Property Testing and Geometry,” HNI-Verlagsschriftenreihe, 119, 2003.

${ }^{\dagger}$ Research supported in part by NSF grants ITR-CCR-0313219 and CCR-0105701, and by DFG grant Me 872/8-2.
} 


\section{Introduction}

The minimum spanning tree problem in graphs belongs to one of the most fundamental problems in algorithmic graph theory. In this paper we study a relaxation of the decision version of this problem for the class of geometric (Euclidean) graphs. We investigate the problem of testing whether a given graph $G$ is a minimum spanning tree for a set of points $P$ in the plane or it is far from any minimum spanning tree for $P$.

We consider a set $P$ of $n$ points in the Euclidean plane $\mathbb{R}^{2}$ and a geometric graph $G=(P, E)$ with vertex set $\mathrm{P}$ and edge set $\mathrm{E}$. Graph $\mathrm{G}=(\mathrm{P}, \mathrm{E})$ is called a Euclidean minimum spanning tree (EMST) of point set $\mathrm{P}$ if $\mathrm{G}$ is a minimum spanning tree of the complete Euclidean graph of $\mathrm{P}$. The complete Euclidean graph is a complete weighted graph, where each edge $e=(p, q) \in P \times P$ has weight equal to the Euclidean distance between $p$ and $q$. For simplicity, we make a standard assumption in computational geometry that $P$ is in general position, i.e., all edge weights are distinct. In this case it is known that the EMST is unique (see, e.g., [8]). We assume that $\mathrm{G}$ is given in adjacency list representation and the set $\mathrm{P}$ is given in an array.

The main result of this paper is a property testing algorithm that for a given $P$ and $G$ accepts the input if $G$ is the EMST of $P$ and that rejects with probability at least $\frac{2}{3}$ every graph $G$ that differs from the EMST of $P$ in more than $\epsilon n$ edges. Our algorithm runs in $\mathcal{O}\left(\sqrt{n / \epsilon} \cdot \log ^{2}(n / \epsilon)\right)$ time and has query complexity of $\mathcal{O}(\sqrt{n / \epsilon} \cdot \log (n / \epsilon))$.

Notice that since the complexity of finding the EMST for a set of $n$ points in $\mathbb{R}^{2}$ is $\Theta(n \log n)$ (even if an approximate solution is sought), our result provides a $\widetilde{\mathcal{O}}(\sqrt{n})$-time, thus sublinear-time approximation procedure to test if a given geometric graph is the EMST.

\subsection{Related research}

Our result lies on the intersection of classical optimization and property testing. We study the Euclidean minimum spanning tree problem and our goal is to approximately verify if a given geometric graph is a minimum spanning tree. Our result follows the framework of property testing [11, 21], which is the computational task to decide if a given object satisfies a certain predetermined property (in our case, input graph is a minimum spanning tree) or is far from every object having this property. If the input object neither has the property nor is far from it, then the algorithm may answer arbitrarily. Thus, the outcome of a property testing algorithm can be seen as an approximation of a property of the input.

The main reason of increasing popularity of property testing in recent years (see, e.g., surveys [9, 10, 20]) is that for a variety of problems the framework of property testing can lead to sublinear-time algorithms, that is, algorithms that can approximately verify if an object has a given property without the need to examine the whole object. This has potential applications in massive data sets and other situations, when even reading the input might be prohibitively expensive. In a sequence of papers, various property testing algorithms have been developed for a variety of problems, starting with graph problems through string problems to problems on matrices, see the survey works [9, 10, 20] and the references therein.

In this paper we present for the first time a property testing algorithm for the minimum spanning tree $(M S T)$ problem. The problem of finding an MST is one of the most fundamental and most extensively studied problems in algorithms. For arbitrary graphs, the currently fastest deterministic algorithm due to Chazelle runs in time $\mathcal{O}(n+m \alpha(m, n))$ [2], where $n$ is the size of the vertex set and $m$ is the number of edges in the input graph (see also [19]). Karger et al. [14] gave a linear-time randomized algorithm. The problem of verifying if the input graph is a minimum spanning tree of another graph in the general case has been investigated in a series of papers [7, 15, 17], where $\mathcal{O}(n+m)$-time algorithms have been given. A better situation is known for Euclidean graphs in $\mathbb{R}^{\mathrm{d}}$. In the case considered in the current paper, that is for $d=2$ (on the plane), Shamos and Hoey [22] gave an $\mathcal{O}(n \log n)$-time algorithm for finding an EMST 
(notice that in this case $m=\Theta\left(n^{2}\right)$ ). We are not aware of any research on the problem of verifying if a Euclidean graph is an EMST for its vertex set

Recently, (after publishing the preliminary version [6] of the current paper) three sublinear-time approximation algorithms have been presented for the problem of estimating the weight of the MST. These algorithms are designed in a similar flavor as that in property testing. Chazelle et al. [3] presented an algorithm that, given a connected graph in adjacency list representation with average degree $\mathrm{D}$, edge weights in the range $[1 \ldots W]$, and a parameter $0<\varepsilon<\frac{1}{2}$, approximates, with high probability, the weight of a MST in time $\widetilde{\mathcal{O}}\left(D W \varepsilon^{-3}\right)$ within a factor of $(1+\varepsilon)$. Czumaj et al. [4] gave a $\widetilde{\mathcal{O}}(\sqrt{n} \cdot \operatorname{poly}(1 / \varepsilon))$-time for a similar problem for geometric graphs, but this algorithm is assuming that the input graph is provided with some additional geometric data structures. Finally, Czumaj and Sohler [5] obtained an $\widetilde{\mathcal{O}}(n \cdot \operatorname{poly}(1 / \varepsilon))$-time algorithm that estimates the weight of the MST in any metric graph to within a factor of $(1+\varepsilon)$.

\subsection{Outline}

After we introduce some basic notation in Section 2 in Section 2.1 we develop a property tester for disjointness of geometric objects, which is used as a subroutine in the EMST tester. Next, in Section 3, we present our property testing algorithm for the EMST problem. Section 4 gives another property testing algorithm which is slightly faster for large values of $1 / \epsilon$.

\section{Preliminaries}

We begin with some basic definitions needed in this section before we discuss the input representation:

Definition 2.1 A geometric graph for $\mathrm{P}$ is a weighted graph $\mathrm{G}=(\mathrm{P}, \mathrm{E})$ with vertex set $\mathrm{P}$ and edge set $\mathrm{E} \subseteq \mathrm{P} \times \mathrm{P}$ (the edges can be interpreted as straight-line segments connecting the endpoints). The weight of an edge $(\mathrm{p}, \mathrm{q})$ is implicitly given by the Euclidean distance between $\mathrm{p}$ and $\mathrm{q}$ in $\mathbb{R}^{2}$.

Definition 2.2 A minimum spanning tree of the complete geometric graph for $\mathrm{P}$ is called the Euclidean minimum spanning tree (EMST) of $\mathrm{P}$.

Next we define when a graph is "far" from the EMST. Typically, a distance measure for graph properties depends on the number of entries in the graph representation that must be changed to obtain a graph that has the tested property.

Definition 2.3 Let $\mathrm{G}=(\mathrm{P}, \mathrm{E})$ be a geometric graph for $\mathrm{P}$ and let $\mathbb{T}=(\mathrm{P}, \mathbb{E})$ be the Euclidean minimum spanning tree of $\mathrm{P}$. We say $\mathrm{G}$ is $\epsilon$-far from being the Euclidean minimum spanning tree of $\mathrm{P}$ (or, in short, $\epsilon$-far from EMST) if one has to modify (insert or delete) more than $\in \mathrm{n}$ edges in $\mathrm{G}$ to obtain $\mathbb{T}$, that is :

$$
|\mathbb{E} \backslash \mathbb{E}|+|\mathbb{E} \backslash \mathbb{E}|>\in \mathfrak{n} .
$$

Input representation. We assume that both the point set and the graph are given as an oracle, and the point set is represented by a function $f:[n] \rightarrow \mathbb{R}^{2}$ (here and throughout the paper we will use the notation $[n]:=\{1, \ldots, n\}$ for the set of integer numbers between 1 and $n)$. The algorithm may query the oracle for the value of $f(i)$ for some $i \in[n]$; the returned value is the position of the $i$ th point of $P$.

The geometric graph is given in the unbounded length adjacency list representation introduced in [18]. The unbounded length adjacency list model is a general model for sparse graphs. The graph structure is 
represented by adjacency lists of varying length. Our property tester may query the degree deg( $p)$ of a vertex $p$ and for each $i \leq \operatorname{deg}(p)$, it may query the ith neighbor of $p$.

The goal of property testing is to develop efficient property testers. A property tester for EMSTs is an algorithm that takes a distance parameter $\epsilon$ and the size $n$ of the input point set $P$ (which equals the number of nodes of $\mathrm{G}$ ). The property tester has oracle access to the function $\mathrm{f}$ representing the point set and to the graph G. A property tester is an algorithm that:

- accepts $\mathrm{G}$, if $\mathrm{G}$ is the EMST of $\mathrm{P}$, and

- rejects $\mathrm{G}$ with probability at least $\frac{2}{3}$, if $\mathrm{G}$ is $\epsilon$-far from EMST.

If $\mathrm{G}$ is neither the EMST nor $\epsilon$-far from it, then the outcome of the algorithm can go either way 1

Complexity of property testers. In this paper we study two types of complexity measures for property testers: the query complexity and the running time. The query complexity measures the number of queries to the oracle asked by a property testing algorithm. If one counts also the time the algorithm needs to perform other tasks than querying the input function values, then the obtained complexity is called the running time of the property tester.

\subsection{Disjointness of a set of objects}

Before we start working on the EMST problem we first consider a simpler problem of disjointness of a set of objects, which will be useful in our analysis of the EMST problem. A set $\mathbb{O}=\left\{\mathrm{O}_{1}, \ldots, \mathrm{O}_{n}\right\}$ of $n$ arbitrary objects is pairwise disjoint if each pair of objects $\mathrm{O}_{i}$ and $\mathrm{O}_{j}$ is disjoint, $1 \leq i<j \leq n$.

Definition 2.4 A set $(\mathbb{O}$ of $n$ objects is $\epsilon$-far from being pairwise disjoint if one has to remove more than $\in \mathrm{n}$ objects from (1) to obtain a pairwise disjoint set of objects.

We prove a simple fact that the following is a property tester for pairwise disjointness of a set of objects.

$$
\begin{aligned}
& \text { DISJOINTNESSTESTER(set of arbitrary objects } \mathbb{O} \text { ) } \\
& \text { Choose a set } S \subseteq \mathbb{O} \text { of size } \mathcal{O}(\sqrt{\mathrm{n} / \epsilon}) \text { uniformly at random } \\
& \text { if } S \text { is disjoint then accept } \\
& \text { else reject }
\end{aligned}
$$

Theorem 1 Algorithm DisJOINTNESSTESTER is a property tester for disjointness of a set of objects. Its query complexity is $\mathcal{O}(\sqrt{\mathrm{n} / \epsilon})$ and its running time is $\mathrm{T}(\mathcal{O}(\sqrt{\mathrm{n} / \epsilon}))+\mathcal{O}(1)$, where $\mathrm{T}(\mathrm{m})$ is the time to decide whether a set of $\mathrm{m}$ input objects is disjoint.

Proof : We have to prove that (1) algorithm DiSJOINTNESSTESTER accepts every set of disjoint objects and (2) that it rejects every set of objects that is $\epsilon$-far from disjoint with probability at least $\frac{2}{3}$.

Part (1) is immediate and thus let us suppose that $\mathbb{O}$ is $\epsilon$-far from disjoint and prove part (2). It is easy to see that we can apply $k=\frac{\epsilon n}{2}$ times the following procedure to $\mathbb{O}$ : pick a pair of intersecting objects $a_{i}$ and $b_{i}, i \in[k]$, and remove it from $\mathbb{O}$. Now, in order to prove that DISJOINTNESSTESTER is a property tester it is sufficient to show that with probability at least $\frac{2}{3}$ at least one of these pairs is in S. We will show only the

\footnotetext{
${ }^{1}$ We consider a one-sided error model, though in the literature also a two-sided error model has been considered, see [9] 10 [20].
} 
bound for the probability of $\frac{1}{4}$; standard amplification techniques can be used to increase the probability to $\frac{2}{3}$ (for example, by increasing the size of $S$ four times).

Using Boole-Bonferroni inequality, straightforward calculations give the following:

$$
\begin{aligned}
\left.\operatorname{Pr}\left[\exists i \in[k]:\left\{a_{i}, b_{i}\right\} \subseteq S\right)\right] & \left.\left.\geq \sum_{i=1}^{k} \operatorname{Pr}\left[\left\{a_{i}, b_{i}\right\} \subseteq S\right)\right]-\sum_{1 \leq i<j \leq k} \operatorname{Pr}\left[\left\{a_{i}, b_{i}, a_{j}, b_{j}\right\} \subseteq S\right)\right] \\
& =\sum_{i=1}^{k} \frac{\left(\begin{array}{c}
n-2 \\
s-2
\end{array}\right)}{\left(\begin{array}{l}
n \\
s
\end{array}\right)}-\sum_{1 \leq i<j \leq k} \frac{\left(\begin{array}{c}
n-4 \\
s-4
\end{array}\right)}{\left(\begin{array}{l}
n \\
s
\end{array}\right)} \geq k\left(\frac{s-1}{n-1}\right)^{2}\left(1-k\left(\frac{s-1}{n-1}\right)^{2}\right) .
\end{aligned}
$$

Hence, $\left.\operatorname{Pr}\left[\exists i \in[k]:\left\{a_{i}, b_{i}\right\} \subseteq S\right)\right] \geq \frac{1}{4}$ for some $s=\Theta(\sqrt{n / \epsilon})$.

\section{Testing Euclidean minimum spanning trees}

We begin with discussion about some basic properties of Euclidean minimum spanning trees. A straightline embedding of a geometric graph is a drawing of the graph on the plane such that all edges are drawn as straight lines; it is crossing-free if its edges do not cross. The following simple claim is well known (see, e.g., [8] 16]):

Claim 3.1 Every Euclidean minimum spanning tree of a point set in general position in $\mathbb{R}^{2}$ is connected, its straight-line embedding is crossing-free and it has maximum degree less than or equal to five.

Now we want to introduce some additional notation that will be useful to simplify the description of the algorithm and its analysis.

Definition 3.2 For a given point set $\mathrm{P}$, a geometric graph $\mathrm{G}=(\mathrm{P}, \mathrm{E})$, and the Euclidean minimum spanning tree $\mathbb{T}=(\mathrm{P}, \mathbb{E})$ of $\mathrm{P}$, the EMST-completion of $\mathrm{G}$ is the geometric graph $\mathrm{G}_{\mathrm{C}}=(\mathrm{P}, \mathrm{E} \cup \mathbb{E})$ that contains all edges that are in $\mathrm{G}$ or in $\mathbb{T}$.

In the next subsection we will present a property tester for EMST that works for a special class of input graphs which we call well-shaped. The restriction to well-shaped graphs simplifies the analysis of the algorithm and it allows a clear view on the important features of the property tester.

Definition 3.3 We call a geometric graph $\mathrm{G}$ well-shaped if

- it is connected,

- the straight-line embedding of its EMST-completion is crossing-free, and

- it has maximum degree at most five.

Notice that by Claim 3.1, if a geometric graph G is the EMST of P then it is well-shaped. Of course, the opposite is not true. Still, however, we can first test if the input graph G is well-shaped and only if it is, we can test if it is the EMST of P. This suggests the following line of the attack: We first test if the input graph is far from a well-shaped graph. If this is the case then we can reject the graph by Claim 3.1 If the input graph passes the test, then we know that (with constant probability) it is either close to a well-shaped graph or it is well-shaped. If the graph is well-shaped we can use the testing algorithm for the special case of well-shaped graphs that will be presented in Sections 3.1 4.1 Then, in Sections 3.3 3.5 we show that we can relax the assumption that the graph is well-shaped and the algorithm will work also for graphs close to well-shaped. This will establish our algorithm. 


\subsection{Properties of EMSTs in well-shaped graphs}

We now design a property tester for EMST when the input graph is well-shaped. First, we give an overview of the algorithm.

Let $\mathrm{G}$ be a well-shaped geometric graph with vertex set $\mathrm{P}$. We first pick a sample set $S \subseteq \mathrm{P}$ using a randomized scheme to be described later. Next, we find the subgraph $G_{S}$ of $G$ that is induced by the vertex set $S$. Then we compute the EMST-completion of $\mathrm{G}_{S}$. If the EMST-completion of $\mathrm{G}$ has a cycle then we reject the input; otherwise we accept.

Now, we proceed with the details. We first show that if the EMST-completion of $\mathrm{G}_{\mathrm{S}}$ contains a cycle then we can always reject the input graph. We use the following lemma which follows easily from standard theory of minimum spanning trees (see, e.g., [23, Chapter 6]). (This lemma uses the fact that the EMST of $P$ is unique.)

Lemma 3.4 Let $\mathrm{S} \subseteq \mathrm{P}$ be a subset of $\mathrm{P}$ and let $\mathrm{p}, \mathrm{q} \in \mathrm{S}$. If the edge $\mathrm{e}=(\mathrm{p}, \mathrm{q})$ does not belong to the EMST of S, then e does not belong to the EMST of $\mathrm{P}$.

Proof : The proof is by contradiction. Let us suppose that $e$ does not belong to the EMST of $S$ and $e$ belongs to the EMST $\mathbb{T}$ of $P$. The removal of $e$ in $\mathbb{T}$ splits $\mathbb{T}$ into two trees. These two trees induce a partition of $\mathrm{P}$ into two subsets $\mathrm{P}_{1}$ and $\mathrm{P}_{2}$. Since $e$ belongs to the EMST of $\mathrm{P}, \mathrm{e}$ must also be the shortest edge between these two subsets. Let $S_{1}=P_{1} \cap S$ and $S_{2}=P_{2} \cap S$. $P_{1}$ and $P_{2}$ are not empty since one vertex of $e$ is in each of the sets. Then $e$ is also the shortest edge between $S_{1}$ and $S_{2}$ and therefore it belongs to the EMST of S; contradiction.

The following are two immediate consequences of Lemma 3.4 that we will use later in the paper.

Corollary 3.5 Let $\mathrm{G}$ be a geometric graph for $\mathrm{P}$. Let $\mathrm{S} \subseteq \mathrm{P}$ and let $\mathrm{G}_{\mathrm{S}}$ be the subgraph of $\mathrm{G}$ induced by $\mathrm{S}$.

- If the EMST-completion $\mathrm{G}^{\prime}=\left(\mathrm{P}, \mathrm{E}^{\prime}\right)$ of $\mathrm{G}$ contains a cycle $\mathrm{Z} \mathrm{C}=\left(\mathrm{p}_{0}, \ldots, \mathrm{p}_{\mathrm{k}}\right)$ of length $\mathrm{k} \geq 3$ with $p_{i} \in \mathrm{S}$ for all $0 \leq i \leq k$, then there is a cycle in the EMST-completion of $\mathrm{G}_{\mathrm{S}}$.

- If the EMST-completion of $\mathrm{G}_{\mathrm{S}}$ contains a cycle $\mathrm{C}=\left(\mathrm{p}_{0}, \ldots, \mathrm{p}_{\mathrm{k}}\right)$ of length $\mathrm{k} \geq 3$, then $\mathrm{G}$ is not the EMST of $\mathrm{P}$.

Now let us consider an input graph $G=(P, E)$ that is $\epsilon$-far from EMST. Our goal is to design a randomized sampling scheme such that the EMST-completion of the subgraph of $\mathrm{G}$ induced by the sample set contains a cycle with high probability. Let $\mathbb{T}=(P, \mathbb{E})$ be the EMST of $P$ and let $G_{C}=(P, \mathbb{E} \cup E)$ be the EMST-completion of G. In the following we refer to the edges in $\mathrm{E}$ as blue edges and to the edges in $\mathbb{E} \backslash E$ as red edges. (Notice a fundamental difference between blue and red edges, in that red edges are given implicitly only, since they do not belong to the input graph G.) We will show that in our analysis, it is sufficient to focus on "short" cycles that contain at most two red edges.

Definition 3.6 Let $\mathrm{C}$ be a cycle of length $\mathrm{k}$ in the EMST-completion of $\mathrm{G}$. We call $\mathrm{C} \epsilon$-short if (1) it is of length $\mathrm{k}$, where $\mathrm{k} \leq \frac{72}{\epsilon}$ and (2) it contains at most two red edges.

\footnotetext{
${ }^{2}$ Here, $C=\left(p_{0}, \ldots, p_{k}\right)$ is a cycle (of length $\left.k\right)$ if $p_{i} \in P$ for all $i \in[k], p_{0}=p_{k},\left(p_{i-1}, p_{i}\right) \in E^{\prime}$ for all $i \in[k]$ and $p_{i} \neq p_{j}$ for all $i, j \in[k], i \neq j$.
} 
In our algorithm we try to find $\epsilon$-short cycles that satisfy some additional "topological" properties. We will exploit the fact that $\mathrm{G}$ is well-shaped, in particular, that the EMST-completion of G has a crossing-free straight-line embedding. Hence we will use a topological representation of the geometric graph $\mathrm{G}$ to exploit the fact that every minimal cycle in a (well-shaped) planar geometric graph corresponds to a face in its straight-line embedding. In order to use this approach in a formal framework we will consider the geometric graph G not only as an undirected graph, but at the same time also using its "directed" representation by "replacing" each undirected edge $(p, q)$ by two directed edges $[p, q\rangle$ and $[q, p\rangle$.
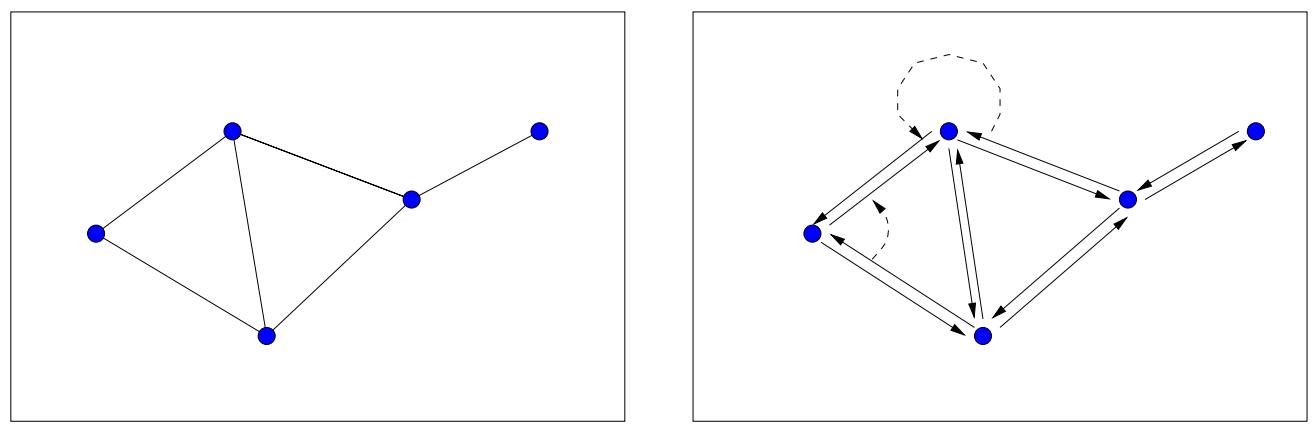

Figure 1: A straight-line embedding and its planar map representation.

For every vertex $p$ in $\mathrm{G}$ we (cyclically) sort incident outgoing edges in clockwise order around the vertex $p$ with respect to the Euclidean positions of the edges' endpoints. (This sorting is done only implicitly, but since we assume that each vertex has a constant degree - at most five, each time we consider a vertex we can sort its incident edges in constant time.) The successor of a directed edge $[p, q\rangle$ is the edge $[q, r\rangle$ where $r$ is the vertex adjacent to $q$ that precedes $p$ in the adjacency list of $q$ (sorted in clockwise order around $q$ ); if $q$ has degree one, then $r=p$. Furthermore, for an edge $e=[p, q\rangle$ in $G$ the kth successor, $k \geq 0$, is defined recursively as follows: the Oth successor of $[p, q\rangle$ is $[p, q\rangle$ itself, and for $k>0$, the kth successor of $[p, q\rangle$ is the successor of the $(k-1)$ st successor of $[p, q\rangle$. Similarly, edge $e$ is the predecessor of edge $e^{\prime}$ if edge $e^{\prime}$ is the successor of edge $e$, and $e$ is the kth predecessor of $e^{\prime}$ if $e^{\prime}$ is the kth successor of $e$. With these definitions, cycles of successive edges (with the exception of cycles of length two, which are not considered as cycles in our analysi 3 ) correspond to faces of the straight-line embedding. Such a representation of $G$ is called a planar map for the straight-line embedding of G (see Figure 1 . We denote it $\widetilde{G}$.

We have introduced the planar map representation of a graph $G$ because it describes the faces of the corresponding embedding in a simple way (using successive edges in $\widetilde{G}$ ). We observe that the correspondence between the faces in the embedding of $G$ and the cycles of successive edges in $\widetilde{G}$ is one to one. We also note that each (directed) edge is contained in exactly one cycle of successive edges. However, a vertex may occur more than once in such a cycle (for an example, see the (inner) boundary of the outer face in Figure 11.

Let $G$ be a well-shaped geometric graph for $P$ and let $C=\left(p_{0}, \ldots, p_{k}\right), k \geq 3$, be a cycle in the planar map of the EMST-completion of G (let us remind that cycles of length two are not considered as cycles). Then $C$ is called topological if for every two consecutive edges on the cycle $\left[p_{i}, p_{i+1}\right\rangle$ and $\left[p_{i+1}, p_{i+2}\right\rangle$, edge $\left[p_{i+1}, p_{i+2}\right\rangle$ is the successor of $\left[p_{i}, p_{i+1}\right\rangle$. We also call the corresponding cycle in G topological.

The following key lemma shows that every well-shaped geometric graph that is far from EMST must

\footnotetext{
${ }^{3} \mathrm{~A}$ cycle of length two corresponds in $\mathrm{G}$ to a single edge incident to a vertex of degree one, and thus, in the planar map representation of G, it consist of two directed edges incident to a vertex of degree one in the straight-line embedding of G.
} 
contain many short topological cycles in its EMST-completion.

Lemma 3.7 Let $\mathrm{G}=(\mathrm{P}, \mathrm{E})$ be a well-shaped geometric graph for $\mathrm{P}$. If $\mathrm{G}$ is $\epsilon$-far from EMST, then there are at least $\frac{\epsilon \mathfrak{n}}{100} \in$-short topological cycles in the EMST-completion of $\mathrm{G}$.

Proof : Let $\mathbb{T}=(P, \mathbb{E})$ be the EMST of P. Let $E_{B}=E \backslash \mathbb{E}$ denote the set of blue edges in the EMSTcompletion of $G$ that are not in the Euclidean minimum spanning tree and let $E_{R}=\mathbb{E} \backslash E$ denote the red edges in the EMST-completion of $G$. Notice that the connectivity of $G$ implies that $\left|E_{B}\right| \geq\left|E_{R}\right|$. Since $G$ is $\epsilon$-far from EMST, we have $\left|E_{B}\right|+\left|E_{R}\right|>\epsilon n$ by definition.

Next, let $\mathrm{H}$ denote the EMST-completion of $\mathrm{G}$ and let $\widetilde{\mathrm{H}}$ denote the planar map of its straight-line embedding. Let $\rho$ be the number of faces in the straight-line embedding of $H$. Then, $\widetilde{H}$ has $\rho$ disjoint topological cycles since each face is bounded by a unique cycle of successive edges. Since $\mathrm{H}$ is planar, connected, and has more than $n-1+\frac{\epsilon \mathfrak{n}}{2}$ edges, we apply Euler's formula to deduce that $\rho \geq \frac{\epsilon n}{2}$.

Now, for every face $f$ in $H$, let $s(f)$ denote the number of (directed) edges in the topological cycle bounding face $f$. Since by Euler's formula $\sum_{f} s(f) \leq 6 n$, there can be at most $\frac{\epsilon n}{8} \leq \frac{\rho}{4}$ faces $f$ with $s(f) \geq \frac{48}{\epsilon}$. Therefore, there are at least $\frac{3 \rho}{4}$ faces $f$ with $s(f)<\frac{48}{\epsilon}$.

Since $\left|E_{R}\right| \leq \rho$, the number of directed red edges is at most $2 \rho$. Hence, the number of topological cycles with 3 or more red edges can be at most $\frac{2 \rho}{3}$. Since we have shown that there are at least $\frac{3 \rho}{4}$ topological cycles having less than $\frac{48}{\epsilon}$ edges, at least $\frac{\rho}{12} \geq \frac{\epsilon n}{24}$ of them have at most two red edges.

Let $\mathrm{G}$ be a well-shaped geometric graph for $\mathrm{P}$. For every vertex $p \in \mathrm{P}$, we define its topological kneighborhood as the set of vertices that are the endpoints of the edges that are either the ith successor of any edge incident to $p, 0 \leq i \leq k$, or the $j$ th predecessor of any edge incident to $p, 0 \leq j \leq k$. The topological $k$-neighborhood of a vertex $p$ is denoted $\mathcal{N}_{\mathrm{G}}^{\text {top }}(p, k)$.

The following claim follows from the fact that every well-shaped graph has maximum degree at most five.

Claim 3.8 Let $\mathrm{G}$ be a well-shaped geometric graph for $\mathrm{P}$. For every vertex $\mathrm{p} \in \mathrm{P}$, we can find its topological $\mathrm{k}$-neighborhood in time $\mathcal{O}(\mathrm{k})$.

\subsection{Property tester in well-shaped graphs}

Now we are ready to present our first property tester for testing if the input well-shaped graph is the EMST of a given input point set. Later, in Section 4.1 in Lemma 4.6 we will present a more complex algorithm that has the query complexity $\mathcal{O}(\log (n / \epsilon) \sqrt{n / \epsilon})$, as compared to the query complexity of $\mathcal{O}\left(\epsilon^{-1} \sqrt{n / \epsilon}\right)$ of the algorithm discussed in this section.

Our approach is to sample uniformly at random a sufficiently large set $Q$ of points in $\mathrm{P}$. Then we add to the sample set the topological $\frac{72}{\epsilon}$-neighborhood of every point in Q. Provided that the set $\mathrm{Q}$ is sufficiently large, we prove in Lemma 3.12 that if $\mathrm{G}$ is $\epsilon$-far from EMST, then the obtained set of vertices will contain a certain $\epsilon$-short topological cycle in the EMST-completion of $\mathrm{G}$ with probability at least $\frac{2}{3}$. By Corollary 3.5. this would certify that $G$ is not an EMST.

We assume that $\mathrm{G}$ is well-shaped. Notice that every $\epsilon$-short topological cycle either

1. is a cycle consisting of at most $\frac{72}{\epsilon}$ blue edges, or

2. is a path consisting of at most $\frac{72}{\epsilon}$ blue edges whose two endpoints are connected by a red edge, or 
3. is a path consisting of at most $\frac{72}{\epsilon}$ blue edges whose two endpoints are connected by a path consisting of exactly two red edges, or

4. consists of two paths containing at most $\frac{72}{\epsilon}$ blue edges whose endpoints are connected to each other by two red edges.

We first observe that if there are many $\epsilon$-short topological cycles of type (1) or (2), then we can easily spot them.

Lemma 3.9 Let $\mathrm{G}=(\mathrm{P}, \mathrm{E})$ be a well-shaped geometric graph. If the EMST-completion of $\mathrm{G}$ contains at least $\frac{\epsilon \mathfrak{n}}{200} \in$-short topological cycles of type (1) or (2), then a set $\mathrm{Q} \subseteq \mathrm{P}$ of size $\frac{4000}{\epsilon}$ chosen uniformly at random with probability at least $\frac{2}{3}$ contains at least one vertex from an $\epsilon$-short topological cycle.

Proof : Since G is well-shaped, it has a maximum degree of five and therefore the EMST-completion of $G$ has maximum degree at most 10 . Thus, every vertex $p \in P$ is contained in at most $10 \epsilon$-short topological cycles. This implies that the set $\mathrm{P}_{\mathrm{C}}$ of all vertices that are contained in at least one $\epsilon$-short topological cycle (of type (1) or (2)) has cardinality at least $\frac{\epsilon n}{2000}$. Now, if we choose a set $Q \subseteq P$ of size $\frac{4000}{\epsilon}$ taken uniformly at random from $P$, then

$$
\operatorname{Pr}\left[\mathrm{Q} \cap \mathrm{P}_{\mathrm{C}}=\emptyset\right] \leq\left(1-\frac{\left|\mathrm{P}_{\mathrm{C}}\right|}{\mathrm{n}}\right)^{|\mathrm{Q}|} \leq\left(1-\frac{\epsilon}{2000}\right)^{|\mathrm{Q}|} \leq \frac{1}{3}
$$

Therefore,

$$
\operatorname{Pr}\left[Q \cap P_{C} \neq \emptyset\right] \geq \frac{2}{3}
$$

Next, we observe that for any $\epsilon$-short topological cycle $C$ of type (1) or (2), for every vertex $v$ from $C$ all other vertices from $C$ belong to the topological $\frac{72}{\epsilon}$-neighborhood of $v$. This motivates us to define the sample set $S$ as the topological $\frac{72}{\epsilon}$-neighborhood of all vertices in $\mathrm{Q}$. Since the set $\mathrm{Q}$ contains at least one vertex from any $\epsilon$-short topological cycle of type (1) or (2) with probability at least $\frac{2}{3}$, we can conclude that $S$ contains all vertices from a particular $\epsilon$-short topological cycle of type (1) or (2) with probability at least $\frac{2}{3}$. Since every vertex of the topological cycle is contained in our sample set we know by Corollary 3.5 that the EMST-completion of the subgraph induced by our sample contains a cycle. Thus our property tester rejects the input with probability $\frac{2}{3}$ if it is $\epsilon$-far from EMST and its EMST-completion contains at least $\frac{\epsilon \mathfrak{n}}{200}$ $\epsilon$-short topological cycles of type (1) or (2).

We can summarize our discussion above in the following lemma.

Lemma 3.10 Let $\mathrm{G}=(\mathrm{P}, \mathrm{E})$ be a well-shaped geometric graph and let $\mathrm{Q} \subseteq \mathrm{P}$ be a set of size $\frac{4000}{\epsilon}$ chosen uniformly at random from $\mathrm{P}$. If the EMST-completion of $\mathrm{G}$ contains at least $\frac{\epsilon \mathfrak{n}}{200} \in$-short topological cycles of type (1) or (2), then the set

$$
S=\bigcup_{p \in Q} \mathcal{N}_{\mathrm{G}}^{\text {top }}\left(p, \frac{72}{\epsilon}\right)
$$

contains all vertices of at least one $\epsilon$-short topological cycle with probability at least $\frac{2}{3}$. 
The $\epsilon$-short topological cycles of type (3) and (4) are more difficult to detect. However, we can still use a very similar approach as for cycles of type (1) or (2), but this time we must find two vertices that belong to the same $\epsilon$-short topological cycle. Suppose that in the EMST-completion of G there are at least $\frac{\epsilon n}{200} \epsilon$-short topological cycles of type (3) or (4). As before, we first take a random subset $\mathrm{Q}$ of $\mathrm{P}$, but this time the size of $Q$ is $\Theta(\sqrt{n / \epsilon})$. Then, we define the sample set $S$ to be the union of the topological $\frac{72}{\epsilon}$-neighborhood of all vertices in $Q$. We show now that the so defined sample set is sufficient to certify that $G$ (if it is $\epsilon$-far from EMST) is not an EMST by proving an analogous statement to Lemma 3.10 for cycles of type (3) and (4):

Lemma 3.11 Let $\mathrm{G}=(\mathrm{P}, \mathrm{E})$ be a well-shaped geometric graph and let $\mathrm{Q} \subseteq \mathrm{P}$ be a set of size $80 \sqrt{\mathrm{n} / \epsilon}$ chosen uniformly at random from $\mathrm{P}$. If the EMST-completion of $\mathrm{G}$ contains at least $\frac{\epsilon \mathfrak{n}}{200} \in$-short topological cycles of type (3) or (4), then the set

$$
\mathrm{S}=\bigcup_{p \in \mathrm{Q}} \mathcal{N}_{\mathrm{G}}^{\mathrm{top}}\left(p, \frac{72}{\epsilon}\right)
$$

contains all vertices of at least one $€$-short topological cycle with probability at least $\frac{2}{3}$.

Proof : For every $\epsilon$-short topological cycle $C$ of type (3), let us define the set $W_{C}$ to contain two vertices: one vertex on the blue path in $C$ and the vertex incident to the two red edges in $C$. Similarly, for every $\epsilon$-short topological cycle $C$ of type (4), let us define the set $W_{C}$ to contain pairs of vertices: one vertex from the first blue path in $\mathrm{C}$ and one vertex from the second blue path in $\mathrm{C}$.

Since each vertex $p \in P$ belongs to at most $10 \epsilon$-short topological cycles, we can select from the sets $W_{C}$ the sets $W_{i}, 1 \leq i \leq \frac{\epsilon \mathfrak{n}}{2000}$, such that the sets $W_{i}$ are disjoint and for each $i, 1 \leq i \leq \frac{\epsilon \mathfrak{n}}{2000}$, there is an $\epsilon$-short topological cycle $C$ with $W_{i}=W_{C}$. Next, we can use the same arguments as in the proof of Theorem 1 to obtain that

$$
\operatorname{Pr}\left[\exists j \in[k]:\left(W_{j} \subseteq Q\right)\right] \geq \frac{2}{3} .
$$

Finally, we observe that if $W_{C} \subseteq \mathrm{Q}$ then all vertices of a cycle $\mathrm{C}$ are in $\mathrm{S}$. Therefore, the lemma follows.

Now we are ready to prove that the following algorithm is a property tester for EMST:

$$
\begin{aligned}
& \text { EMST-TEST-SIMPLE }(G, \epsilon) \\
& \mathrm{s}=80 \sqrt{\mathrm{n} / \epsilon}+4000 / \epsilon \\
& \text { choose a set } \mathrm{Q} \subseteq \mathrm{P} \text { of size s uniformly at random } \\
& \mathrm{S}=\bigcup_{\mathrm{q} \in \mathrm{Q}} \mathcal{N}_{\mathrm{G}}^{\mathrm{top}}\left(\mathrm{q}, \frac{72}{\epsilon}\right) \\
& \text { compute the subgraph } \mathrm{G}_{\mathrm{S}} \text { induced by } \mathrm{S} \\
& \text { compute the EMST-completion } \mathrm{G}_{\mathrm{C}} \text { of } \mathrm{G}_{\mathrm{S}} \\
& \text { if } \mathrm{G}_{\mathrm{C}} \text { contains a cycle then reject } \\
& \text { else accept }
\end{aligned}
$$

Lemma 3.12 Let $\mathrm{G}$ be a well-shaped geometric graph for $\mathrm{P}$. Then there is a property tester that in time $\mathcal{O}\left(\sqrt{n / \epsilon^{3}} \cdot \log (n / \epsilon)\right)$ and with query complexity $\mathcal{O}\left(\sqrt{n / \epsilon^{3}}\right)$ accepts the input if $\mathrm{G}$ is an EMST of $\mathrm{P}$ and rejects the input with probability at least $\frac{2}{3}$ if $\mathrm{G}$ is $\epsilon$-far from EMST. 
Proof: By Corollary 3.5 if the input graph $\mathrm{G}=(\mathrm{P}, \mathrm{E})$ is the EMST then EMST-TEST-SIMPLE accepts.

Now let us consider the case when G is $\epsilon$-far from EMST. Then, by Lemma 3.7 we know that there are $\frac{\epsilon \mathfrak{n}}{100} \epsilon$-short topological cycles in the EMST completion of G. It follows that there are $\frac{\epsilon \mathfrak{n}}{200}$ cycles of type (1) and (2) or $\frac{\epsilon \mathfrak{n}}{200}$ cycles of type (3) or (4). By Lemma 3.10 and 3.11 we know that the sample taken by EMST-TEST-SIMPLE contains an $\epsilon$-short topological cycle with probability at least $\frac{2}{3}$. By Corollary 3.5 we know that then there is a cycle in the EMST-completion of the subgraph induced by our sample. Hence the algorithm rejects in such a case.

The query complexity of the algorithm is immediate. Its running time follows from Claim 3.8 and the fact that the EMST completion of a graph with $m$ vertices can be computed in time $\mathcal{O}(\mathrm{m} \log \mathrm{m})$.

\subsection{Testing connectivity and crossing-freeness of EMST-completions in bounded degree graphs}

Now we want to relax the condition that the input graphs are well-shaped. We develop property testers for connectivity and crossing-free EMST-completions in graphs of maximum degree at most five. If for a given set of points $P$ the input graph $G$ of maximum degree at most five is an EMST then the tester accepts G. If however $G$ is $\epsilon$-far from connected or the straight-line embedding of the EMST-completion of $G$ is $\epsilon$-far from crossing-free, respectively, then the tester rejects $G$ with probability at least $\frac{2}{3}$.

\subsubsection{Testing connectivity in bounded degree graphs}

We begin with a test if a graph of maximum degree at most five is connected. We say a geometric graph $\mathrm{G}$ for $\mathrm{P}$ is $\epsilon$-far from connected if one has to add more than $\epsilon \mathrm{n}$ edges to $\mathrm{G}$ to obtain a connected graph. Observe that this definition if equivalent to saying that $\mathrm{G}$ has more than $€ n+1$ connected components.

Since the property of being connected does not depend on the positions of the input points in $\mathrm{P}$, we can use a property tester for connectivity in graphs.

Lemma 3.13 [12] Let $\mathrm{G}$ be a graph with degree bound $\mathrm{d}$. Connectivity of $\mathrm{G}$ in the bounded length adjacency list mode 4 can be tested with $\mathcal{O}\left(\frac{\log ^{2}(1 / \epsilon \mathrm{d})}{\epsilon \mathrm{d}}\right)$ time and query complexity.

We can immediately apply this result to geometric graphs:

Corollary 3.14 Let $\mathrm{G}$ be a geometric graph for $\mathrm{P}$ with maximum degree five. There is a property tester that in time $\mathcal{O}\left(\frac{\log ^{2}(1 / \epsilon)}{\epsilon}\right)$ and with a query complexity of $\mathcal{O}\left(\frac{\log ^{2}(1 / \epsilon)}{\epsilon}\right)$ accepts the input if $\mathrm{G}$ is connected and rejects the input with probability at least $\frac{2}{3}$ if $\mathrm{G}$ is $\epsilon$-far from connected.

Proof : We run the tester from [12] with $d=5$ and $\epsilon^{\prime}=\frac{\epsilon}{5}$.

\subsubsection{Testing crossing-free EMST-completions in bounded degree graphs}

Next, we design a property tester that accepts the input graph (with maximum degree at most five) if it is the EMST and rejects it if the straight-line embedding of its EMST-completion is $\epsilon$-far from crossing-free. We

\footnotetext{
${ }^{4}$ In the bounded degree graph model a graph is $\epsilon$-far from connected if one has to add more than $\epsilon \mathrm{d} n$ edges to obtain a connected graph.
} 
say that the straight-line embedding of a geometric graph $\mathrm{G}$ for $\mathrm{P}$ is $\epsilon$-far from crossing-free if one has to remove more than $€ n$ edges in $\mathrm{G}$ to obtain a crossing-free straight-line embedding of its EMST completion.

We proceed in two steps. First, our property tester checks for pairs of intersecting blue edges and then for intersections between blue and red edges; red edges cannot intersect because they are edges of the EMST.

We first use the tester DisJOINTNESSTESTER developed in Section 2.1 to find intersections between blue edges. Since $\mathrm{G}$ has maximum degree five, it has at most $2.5 \mathrm{n}$ edges. Therefore, since one can verify in time $\mathcal{O}(n \log n)$ if a geometric graph with $n$ vertices has crossing-free straight-line embedding [1], Theorem 1 implies the following result.

Lemma 3.15 Let $\mathrm{G}$ be a geometric graph with maximum degree at most five. There is a property tester that in time $\mathcal{O}(\sqrt{n / \epsilon} \log (n / \epsilon))$ and with the query complexity of $\mathcal{O}(\sqrt{n / \epsilon})$ accepts the input if the straightline embedding of $\mathrm{G}$ is crossing-free and rejects the input with probability at least $\frac{2}{3}$ if the straight-line embedding of $\mathrm{G}$ is $\epsilon$-far from crossing-free.

It remains to design a property tester for red-blue intersections in the EMST-completion of G. (More precisely, we do not design a property tester for the property of having no red-blue intersections. Our algorithm might reject an input graph if its EMST-completion has no red-blue intersections. However, if the input graph is the EMST then it is always accepted by our algorithm.) A geometric graph with red and blue edges has a straight-line embedding without red-blue intersections if there is no intersection between the corresponding red and blue segments. Similarly, the straight-line embedding of a geometric graph whose edges are colored blue and red is $\epsilon$-far from having no red-blue intersections if one has to delete more than an $\epsilon$-fraction of its edges to remove all red-blue intersections.

The main difficulty with testing for red-blue intersections in the EMST-completion of G is caused by the fact that the red edges are defined only implicitly, because they do not belong to the input graph G. We will use the following lemma to study properties of intersections of explicitly given blue edges with implicitly given red ones.

Lemma 3.16 Let $\overline{\mathrm{pq}}$ be a red and $\overline{\mathrm{xy}}$ be a blue segment in the EMST-completion of $\mathrm{G}$. If $\overline{\mathrm{pq}}$ and $\overline{\mathrm{xy}}$ intersect each other, then either edge $(\mathrm{x}, \mathrm{y})$ is not in the EMST of any set containing $\{x, y, p\}$, or $(x, y)$ is not in the EMST of every set containing $\{x, y, q\}$.

Proof: The points $p, q, x, y$ are in convex position because the segments $\overline{p q}$ and $\overline{x y}$ intersect. We consider the quadrilateral pxqy (see Figure 2). Let us call the inner angles in the quadrilateral at vertices $p, q, x, y$ to be $\alpha, \beta, \gamma$, and $\delta$, respectively. Let us recall that the longest edge of a triangle is opposite of the largest angle.

If $\alpha<\frac{\pi}{2}$ and $\beta<\frac{\pi}{2}$ then $\gamma$ or $\delta$ is larger than $\frac{\pi}{2}$ because $\alpha+\beta+\gamma+\delta=2 \pi$. Without loss of generality, let $\gamma>\frac{\pi}{2}$. Then, segment $\overline{p q}$ is the longest edge of triangle $p q x$ and thus it cannot be the EMST of $\{p, q, x\}$. By Lemma 3.4 this is a contradiction to the fact that $(p, q)$ is an edge of the EMST. Hence we must have either $\alpha \geq \frac{\pi}{2}$ or $\beta \geq \frac{\pi}{2}$.

If $\alpha \geq \frac{\pi}{2}$ then segment $\overline{x y}$ is the longest edge in triangle pxy. Hence edge $(x, y)$ is not contained in the EMST of $p, x, y$. By Lemma 3.4 it is also not contained in any EMST of a subset of $P$ that contains $p, x, y$. Similarly, if $\beta \geq \frac{\pi}{2}$ then edge $(x, y)$ is not contained in any EMST of a subset of $P$ that contains $q, x, y$.

This lemma shows that each red-blue intersection between a red edge $(p, q)$ and a blue edge $(x, y)$ has a "witness" consisting of one point $z \in\{p, q\}$ and the edge $(x, y)$ so that $(x, y)$ is not in the EMST of $x, y, z$.

Our property tester for red-blue intersections is similar to the DISJOINTNESSTESTER tester but we use a modified definition of disjointness property: We say that two points $v, u \in P$ intersect if there is a point $w \in P$ such that at least one of $(v, w)$ and $(u, w)$ is a blue edge that is not in the EMST of $v, u, w$. 


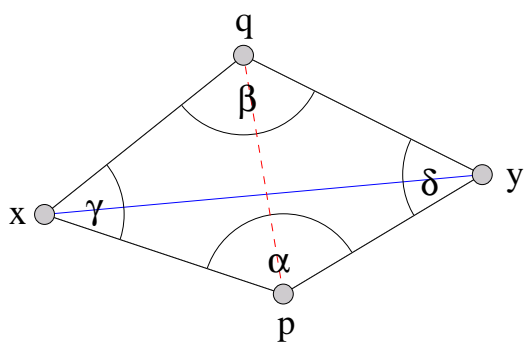

Figure 2: A quadrilateral $\langle p, q, x, y\rangle$ with red blue intersection. The red edge is dotted.

We now are ready to present our property tester for red-blue intersections:

$\operatorname{RedBLueTest}(G, \epsilon)$

Choose a set $S^{\prime} \subset P$ of size $16 \sqrt{5 n / \epsilon}$ uniformly at random

Let $S=S^{\prime} \cup \mathcal{N}\left(S^{\prime}\right)$, where $\mathcal{N}\left(S^{\prime}\right)$ denotes the set of neighbors of points in $S^{\prime}$

Let $\mathrm{G}_{S}$ denote the subgraph induced by $S$

if the EMST-completion of $\mathrm{G}_{S}$ has a cycle then reject

else accept

The analysis of the algorithm is similar to the analysis of DisJOINTNESSTESTER, Theorem 1

Lemma 3.17 Let $\mathrm{G}$ be a geometric graph for $\mathrm{P}$ with maximum degree five. Algorithm REDBLUETEST runs in time $\mathcal{O}(\sqrt{n / \epsilon} \log n)$ and with the query complexity of $\mathcal{O}(\sqrt{n / \epsilon})$, and accepts the input graph $\mathrm{G}$ if it is the EMST of $\mathrm{P}$ and rejects the input with probability at least $\frac{2}{3}$ if the straight-line embedding of the EMST-completion of $\mathrm{G}$ is $\epsilon$-far from having no red-blue intersection.

Proof : Obviously, if $\mathrm{G}=(\mathrm{P}, \mathrm{E})$ is the EMST then algorithm RedBLuETeST accepts $\mathrm{G}$.

Let $\mathrm{G}_{C}$ denote the EMST-completion of $\mathrm{G}$ and let us assume that $\mathrm{G}_{C}$ is $\epsilon$-far from having no redblue intersections. By Lemma 3.16 we can apply the following procedure $k=\frac{\epsilon \mathfrak{n}}{20}$ times: pick a pair of intersecting (according to the definition above) points $\{v, u\}=W_{i}, i \in[k]$, and remove all edges incident to $v$ and $u$ from $\mathrm{G}_{\mathrm{C}}$. By the degree bound, we removed at most 10 edges for the two vertices, and therefore this procedure can be performed at least $k$ times.

In order to prove that if $G_{C}$ is $\epsilon$-far from having no red-blue intersections then REDBLUETEST rejects $\mathrm{G}$ with probability at least $\frac{2}{3}$, we first use the same arguments as those used in the proof of Theorem 1 to obtain:

$$
\operatorname{Pr}\left[\exists j \in[k]:\left(W_{j} \subseteq S^{\prime}\right)\right] \geq \frac{2}{3}
$$

It remains to show that if there is a set $W_{i} \subseteq S^{\prime}$ then the algorithm rejects the input graph. If $W_{i}=$ $\{v, u\} \subseteq S^{\prime}$ then there exists a blue edge $e=(v, w)$ (or $e=(u, w)$ ) such that $e$ is not in the EMST of $v, u, w$. Therefore, by Lemma 3.4 e is not in the EMST of S. Hence $S$ has a cycle and RedBlueTest rejects.

Finally, we can combine Lemmas 3.15 and 3.17 to obtain the following result. 
Lemma 3.18 Let $\mathrm{G}$ be a geometric graph for $\mathrm{P}$ with maximum degree five. There is an algorithm that in time $\mathcal{O}(\sqrt{n / \epsilon} \log (\mathrm{n} / \epsilon))$ and with a query complexity of $\mathcal{O}(\sqrt{n / \epsilon})$ accepts $\mathrm{G}$ if $\mathrm{G}$ is the EMST of $\mathrm{P}$ and rejects $\mathrm{G}$ with probability at least $\frac{2}{3}$ if the straight-line embedding of the EMST-completion of $\mathrm{G}$ is $\in$-far from crossing-free.

Proof : Let $G$ be $\epsilon$-far from having a crossing-free EMST-completion. Then, either the straight-line embedding of $\mathrm{G}$ is $\frac{\epsilon}{2}$-far from crossing-free or the EMST-completion of $\mathrm{G}$ is $\frac{\epsilon}{2}$-far from having no red-blue intersections. Applying Lemma 3.15 and Lemma 3.17 with $\epsilon=\frac{\epsilon}{2}$ shows that G is rejected with probability at least $\frac{2}{3}$. Since the tester for blue-blue intersection and the tester for red-blue intersections both accept the EMST, this completes the proof of Lemma 3.18

\subsection{Property tester for low degree}

Our next step is to deal with graphs that have maximum degree greater than five. A graph $G$ is $\epsilon$-far from having low degree if one has to remove more than $\epsilon n$ edges in $\mathrm{G}$ to obtain a graph having maximum degree smaller than or equal to five. In this section, we develop an efficient property tester for low degree.

Let us call a vertex heavy if it either has degree greater than five or it has a neighbor of degree greater than five. Observe that if in a graph we removed all edges between heavy vertices then we would obtain a graph of maximum degree at most five. Therefore, if $\mathrm{G}$ is $\epsilon$-far from having small degree, then there are at least $\sqrt{\epsilon n}$ heavy vertices in G. (Indeed, for if not, then we could remove less than $(\sqrt{\epsilon n})^{2}=\epsilon n$ edges and obtain a graph with maximum degree at most five, what contradicts the assumption that $G$ is $\epsilon$-far from having small degree.) Therefore the simple algorithm that picks a random set $S$ of $4 \sqrt{n / \epsilon}$ points in $P$ and tests if every point $p \in S$ has the degree smaller than or equal to five and if so then it tests if every neighbor of $p \in S$ has degree smaller than or equal to five, will detect with probability greater than or equal to $\frac{2}{3}$ every geometric graph $\mathrm{G}$ that is $\epsilon$-far from having small degree.

Lemma 3.19 Let $\mathrm{G}$ be a geometric graph for $\mathrm{P}$. There is a property tester that in time $\mathcal{O}(\sqrt{\mathrm{n} / \epsilon})$ and with a query complexity of $\mathcal{O}(\sqrt{\mathrm{n} / \epsilon})$ accepts the input if $\mathrm{G}$ has a maximum degree smaller than or equal to five and rejects the input with probability at least $\frac{2}{3}$ if $\mathrm{G}$ is $\in$-far from having small degree.

Proof : Clearly, our algorithm accepts every graph having maximum degree of five. Let us assume that $\mathrm{G}$ is $\epsilon$-far from having small degree. Let $S$ be a sample of size $4 \sqrt{\epsilon n}$ chosen uniformly at random from $P$.

$$
\operatorname{Pr}[S \text { contains no heavy vertex }] \leq(1-1 / \sqrt{n / \epsilon})^{4 \sqrt{n / \epsilon}} \leq \frac{1}{3} .
$$

It follows that

$$
\operatorname{Pr}[S \text { contains a heavy vertex }] \geq \frac{2}{3} .
$$

Hence our algorithm rejects every graph that is $\epsilon$-far from having small degree with probability at least $\frac{2}{3}$. Thus it is a property tester.

The running time and the query complexity follow from the fact that since the degree of every $p \in S$ is less than or equal to five, all operations can be performed in a constant time per vertex $p$. 


\subsection{Property tester for general graphs}

To obtain a tester for general graphs, we first test if the input graph is $\frac{\epsilon}{4}$-far from having low degree (using the tester described in Section 3.4. Then, we run the tester for testing if a graph of maximum degree at most five is $\frac{\epsilon}{4}$-far from being connected (using the tester described in Section 3.3.1. Next, we test if a graph of maximum degree at most five is $\frac{\epsilon}{4}$-far from having straight-line embedding of its EMST-completion crossing-free (using the tester described in Section 3.3.2. Finally, we run our algorithm that tests if a given well-shaped geometric graph is $\frac{\epsilon}{4}$-far EMST. There is one important modification in all these algorithms:

- For each vertex $v \in S$, we also include every neighbor of $v$ in $G$ into the sample set. This can be done without asymptotically increasing the running time of the algorithm (because if we encounter a vertex with degree greater than five then we reject $G$ ).

- If during the course of the algorithm we encounter a vertex with degree greater than five, we immediately reject the input graph.

- If during the course of the algorithm we detect that the input graph is not connected then we immediately reject the input graph.

- If during the course of the algorithm we detect that the straight-line embedding of the EMST-completion of the input graph is not crossing-free then we immediately reject the input graph.

Clearly, the above modifications do not affect the case when the input graph is the EMST of the point set: the algorithm will still accept the input graph. Thus let us consider the case when the input graph G is $\epsilon$-far from EMST. If the low degree tester rejects the input graph, we are done. Thus let us assume that the input graph passes this test but it is $\epsilon$-far from EMST. Now we define the graph $G^{\prime}$ to be a graph obtained from $G$ by deleting a minimal set of edges such that $G^{\prime}$ has maximum degree of five. Since we deleted less than $\frac{\epsilon n}{4}$ edges from $G$ to obtain $G^{\prime}$, we conclude that $G^{\prime}$ is $\frac{3 \epsilon}{4}$-far from EMST.

In order to analyze the behavior of the modified algorithm for testing the three other properties, we consider the (unmodified) algorithm for graphs with maximum degree five. First of all, we observe that if there is a heavy vertex in the sample chosen by the unmodified algorithm then the modified algorithm always rejects. But if there is no heavy vertex in the sample chosen by the unmodified algorithm then the graph "looks" like the graph $G^{\prime}$ which has maximum degree five and is $\frac{3 \epsilon}{4}$-far from EMST.

Once we have an algorithm running on graphs with maximum degree at most five, our testers for connectivity and crossing-freeness of straight-line embedding of the graph's EMST-completion can be used without any further modifications. Therefore, after running these two testers, we can assume, with probability at least $\left(\frac{2}{3}\right)^{3}$, that the input graph has maximum degree at most five, is not $\frac{\epsilon}{4}$-far from being connected and is not $\frac{\epsilon}{4}$-far from having its EMST-completion crossing-free. Next, we observe that since the algorithm did not spot a heavy vertex, nor a disconnected component, nor a crossing in the graph's EMST-completion, for the algorithm from Lemma 3.12 the graph "looks" like the graph $\mathrm{G}^{\prime}$ which is well-shaped. Therefore, if we run the algorithm from Lemma3.12, then it will reject a graph that is $\frac{\epsilon}{4}$-far from EMST with probability $\frac{2}{3}$. Hence, these arguments imply the following main theorem.

Theorem 2 There is a property tester for the EMST property with a running time of $\left.\mathcal{O}\left(\sqrt{n / \epsilon^{3}}\right) \cdot \log (n / \epsilon)\right)$ and with a query complexity of $\mathcal{O}\left(\sqrt{\mathrm{n} / \epsilon^{3}}\right)$.

Finally, we remark that the entire analysis can be carried over to the case of arbitrary (possibly degenerated) inputs, not necessarily in the general position. The main idea is to replace the (unique) minimum 
spanning tree in the analysis for point sets in general position by the minimum spanning tree that is nearest (in terms of edit distance) to the input graph. Our algorithm will also compute such spanning tree on its sample set of points. This can be done by first computing a minimum spanning tree of the sample set. Then we take the union of this minimum spanning tree and the input graph and compute the minimum spanning tree of this union. During this computation we use the following tie-breaker rule for edge lengths: If two edges have the same length and one of them is contained in the input graph, we prefer this edge. This way, we obtain the minimum spanning tree that is closest to the input graph. If the union of this tree with the subgraph of the input graph induced by our sample set has a circle, then we found a counter example and can safely reject.

\section{Another algorithm: stronger bound for large $\epsilon$}

In the previous section, we presented a property tester for the EMST property with a running time of $\left.\mathcal{O}\left(\sqrt{n / \epsilon^{3}}\right) \cdot \log (n / \epsilon)\right)$ and with a query complexity of $\mathcal{O}\left(\sqrt{n / \epsilon^{3}}\right)$. In this section, we present another algorithm whose complexity is better for large values of $\epsilon$; its running time is $\left.\mathcal{O}(\sqrt{n / \epsilon}) \cdot \log ^{2}(n / \epsilon)\right)$ and its query complexity is $\mathcal{O}(\sqrt{n / \epsilon} \cdot \log (n / \epsilon))$. The algorithm differs only in one step: we use another algorithm for testing EMST in well-shaped graphs.

\subsection{Improved property tester in well-shaped graphs}

In this section we present a modification of the property tester $\operatorname{EMST-TEST-SIMPLE}(G, \epsilon)$ that has a slightly better complexity. In our property tester in Section 3.2 we were always trying to catch one initially fixed single vertex from each blue path although an $\epsilon$-short topological cycle can contain as many as $\frac{72}{\epsilon}$ vertices. We now want to take the length of the topological cycles into consideration. Furthermore, we were always taking topological $\frac{72}{\epsilon}$-neighborhoods of all vertices. This strategy should be applied to the cycles that have as many as $\frac{72}{\epsilon}$ edges, but it is not necessary for shorter cycles. Our approach now is to improve the complexity of the property tester by combining these two observations. We show that if the input graph is well-shaped then the following algorithm is a property tester for EMST:

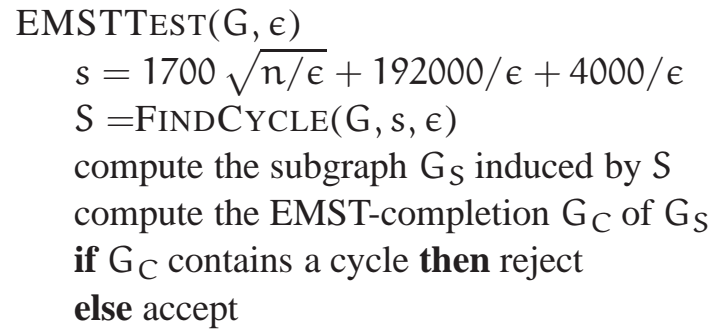

Where the procedure FINDCYCLE is the following: 


$$
\begin{aligned}
& \text { FindCyCLE }(G, s, \epsilon) \\
& \mathcal{S}^{(0)}=\emptyset \\
& \text { for } i=1 \text { to } 2 s \text { do } \\
& j=0 \\
& \text { pick a vertex } p^{(i)} \in P \text { uniformly at random } \\
& \text { while } j \leq \log \frac{72}{\epsilon} \text { do } \\
& j=j+1 \\
& \text { flip a coin } \\
& \text { if head then exit the while-loop } \\
& S^{(i)}=S^{(i-1)} \cup \mathcal{N}_{G}^{\text {top }}\left(p^{(i)}, 2^{j}\right) \\
& \text { return } S^{(2 s)}
\end{aligned}
$$

First, we observe that by Corollary 3.5 algorithm EMSTTEST accepts every EMST. Therefore, we only have to prove that if the input graph is $\epsilon$-far from EMST then it is rejected with probability at least $\frac{2}{3}$.

Let us assume that $G$ is well-shaped and $\epsilon$-far from EMST. Then, by Lemma 3.7 there are at least $\frac{\epsilon n}{100}$ $\epsilon$-short topological cycles in the EMST-completion of G. Let $\mathfrak{C}_{j}, j=1,2,3,4$, denote the set of all $\epsilon$-short topological cycles of type (j) in the EMST-completion of G. Now we consider separately cycles in $\mathfrak{C}_{1} \cup \mathfrak{C}_{2}$ and cycles in $\mathfrak{C}_{3} \cup \mathfrak{C}_{4}$. By our discussion above we have either $\left|\mathfrak{C}_{1} \cup \mathfrak{C}_{2}\right| \geq \frac{\epsilon \mathfrak{n}}{200}$ or $\left|\mathfrak{C}_{3} \cup \mathfrak{C}_{4}\right| \geq \frac{\epsilon \mathfrak{n}}{200}$.

From now on, to simplify the notation, we will use $\lambda=\frac{\epsilon}{200}$.

Cycles of type (1) and (2). Suppose that $\mathrm{G}$ is a geometric graph for $\mathrm{P}$ with maximum degree five and there are at least $\lambda n \in$-short topological cycles of type (1) or (2) in the EMST-completion of G. We first consider the probability that a fixed $\epsilon$-short topological cycle $C \in \mathfrak{C}_{1} \cup \mathfrak{C}_{2}$ is contained in the sample set. Let $\ell$ denote the number of vertices in cycle $C$. Then the probability that in round $i$ of the FINDCYCLE procedure vertex $p^{(i)}$ is one of the $\ell$ vertices of cycle $C$ is $\frac{\ell}{n}$. Furthermore, the probability that the topological neighborhood of $p^{(i)}$ is chosen large enough to contain all vertices of $C$ is at least $\frac{1}{2 \ell}$. Overall, for a fixed cycle $C$ the probability that a vertex of $\mathrm{C}$ is chosen in round $i$ and that the topological neighborhood of the vertex is large enough is at least $\frac{1}{2 n}$. If the cycles are vertex disjoint then it is simple to prove that after $\mathcal{O}\left(\frac{1}{\epsilon}\right)$ rounds at least one cycle is completely contained in the sample set with constant probability. Unfortunately, in the general case the cycles are not vertex disjoint. To overcome this technical problem we use the planar map representation of $G$ and the following trick for the analysis: Instead of taking the whole topological $2^{j}$-neighborhood of vertex $p^{(i)}$ we assume that our algorithm selects only one of the outgoing edges (in its planar map representation) uniformly at random. Then it includes only the $2^{j}$ successors and predecessors of the chosen edge in the planar map representation of G. Clearly, this procedure considers only a subset of the vertices considered in the original procedure. Nevertheless, we can show that the set of vertices we pick using this procedure will contain the vertices of $C$ with sufficiently large probability. For the analysis we use the fact that every cycle corresponds to the boundary of a face in the planar map representation of the EMST completion. Thus, for every cycle $C$ there is a unique directed cycle $C^{\prime}$. Furthermore, these directed cycles are edge disjoint.

Assume that we pick a vertex that belongs to cycle $C^{\prime}$. Provided that $j$ is large enough we still have to choose the correct directed outgoing edge to have all vertices of $C^{\prime}$ (and hence all vertices of $C$ ) in the sample set. Since our graph has a degree bound of five the probability that this edge is chosen is at least $\frac{1}{5}$. Since type (2) cycles with $\ell$ vertices are spanned by a path of (at least) $\ell-1$ (directed) blue edges, the probability that $p^{(i)}$ is one of the origins of these edges is $\frac{\ell-1}{n} \geq \frac{\ell}{2 n}$ (a directed edge points from its origin 
to its destination). Hence the probability that a directed cycle $C^{\prime}$ of type (1) or (2) is completely contained in the sample set is at least $\frac{1}{20 n}$. We know that all directed cycles are disjoint and so the probability that at least one directed cycle is completely contained in the sample set taken in round $i$ is at least $\frac{\lambda n}{20 n}=\frac{\lambda}{20}$. Since each directed cycle $C^{\prime}$ has the same set of vertices as the corresponding undirected cycle $C$ and since the sample set taken in round $i$ by our modified procedure is a subset of the topological $2^{j}$-neighborhood of $p^{(i)}$, the probability that all vertices of cycle $C$ are in the topological $2^{j}$-neighborhood of $p^{(i)}$ is the at least the probability that all vertices of the directed cycle $C^{\prime}$ are in the sample set taken in round $i$ by our modified procedure. Hence, we know that in round $i$ with probability at least $\frac{\lambda}{20}$ for some cycle $C \in \mathfrak{C}_{1} \cup \mathfrak{C}_{2}$ its set of vertices is completely contained in the topological $2^{j}$-neighborhood of $p^{(i)}$. Let $X^{(2 s)}$ denote the indicator random variable for the event that all vertices of some cycle $C \in \mathfrak{C}_{1} \cup \mathfrak{C}_{2}$ are contained in the sample set $S^{(2 s)}$. Then we have for $s \geq \frac{20}{\lambda}=\frac{4000}{\epsilon}$ :

$$
\operatorname{Pr}\left[X^{(2 s)}=0\right] \leq\left(1-\frac{\lambda}{20}\right)^{2 s} \leq \frac{1}{3}
$$

and hence

$$
\operatorname{Pr}\left[X^{(2 s)}=1\right] \geq \frac{2}{3}
$$

and so we have just proved:

Lemma 4.1 Let $\mathrm{G}$ be a geometric graph for $\mathrm{P}$ with maximum degree five that has at least $\frac{\mathrm{\epsilon}}{200}$ topological $\epsilon$-short cycles of type (1) or (2). If algorithm $\operatorname{FindCycle}(\mathrm{G}, s, \epsilon)$ is invoked with $\mathrm{s} \geq \frac{4000}{\epsilon}$ then the set $\mathrm{S}^{(2 \mathrm{~s})}$ returned by the algorithm contains an $\epsilon$-short topological cycle with probability at least $\frac{2}{3}$.

Cycles of type (3) and (4). Let $G$ be a geometric graph with maximum degree five. Let us further assume that there are at least $\lambda n$ topological $\epsilon$-short cycles of type (3) and (4) in the EMST-completion of G, for $\lambda=\frac{\epsilon}{200}$. We show that the sample set computed by algorithm FINDCYCLE contains every vertex of at least one $\epsilon$-short topological cycle with good probability.

Recall that cycles of type (4) consist of 2 paths of blue edges connected by two red edges. Cycles of type (3) are a special case of type (4) cycles: The shorter path has length 0 . For each cycle $C \in \mathfrak{C}_{3} \cup \mathfrak{C}_{4}$ let $X_{C}^{(i)}$ denote the indicator random variable for the event that all vertices of the longer (blue) path of cycle $C$ are in $S^{(i)}$. Let $Y_{C}^{(i)}$ be the indicator random variable for the event that all vertices of the shorter (blue) path of cycle $C$ are in $S^{(i)}$. Furthermore, let $\Delta^{(i+1)}$ be the indicator random variable for the event that there is a cycle $C^{\prime} \in \mathfrak{C}_{3} \cup \mathfrak{C}_{4}$ with $X_{C^{\prime}}^{(i)}=0$ and $X_{C^{\prime}}^{(i+1)}=1$. We say that a cycle $C \in \mathfrak{C}_{3} \cup \mathfrak{C}_{4}$ is half-contained in $S^{(i)}$ if $X_{C}^{(i)}=1$. Cycle $C$ is contained in $S^{(i)}$ if $X_{C}^{(i)}=1$ and $Y_{C}^{(i)}=1$.

We analyze the algorithm in two steps. We first show that with high probability many (at least $\frac{\lambda s}{80}$ ) topological $\epsilon$-short cycles are half-contained in the set $S^{(s)}$. Then we show that the set $S^{(2 s)}$ contains at least one cycle $C \in \mathfrak{C}_{3} \cup \mathfrak{C}_{4}$ with high probability.

Claim 4.2 Let the outcome of the random choices in round 1 to $i$ of the for-loop of FINDCYCLE be fixed. If

$$
\sum_{\mathrm{C} \in \mathfrak{C}_{3} \cup \mathfrak{C}_{4}} \mathrm{X}_{\mathrm{C}}^{(\mathfrak{i})}<\frac{\lambda s}{2}
$$

then

$$
\operatorname{Pr}\left[\Delta^{(i+1)}=1\right] \geq \frac{\lambda}{40}
$$


Proof : Let us assume that (1) holds. Then we observe that:

$$
\sum_{\mathrm{C} \in \mathfrak{C}_{3} \cup \mathfrak{C}_{4}} X_{\mathrm{C}}^{(\mathfrak{i})}<\frac{\lambda s}{2} \leq \frac{\lambda n}{2}
$$

since $s \leq n$. We conclude that we have more than $\frac{\lambda \mathfrak{n}}{2}$ cycles in $\mathfrak{C}_{3} \cup \mathfrak{C}_{4}$ that are not half-contained in $S^{(i)}$. If $p^{(i+1)}$ is one of the vertices of the longer path of one of these cycles and if the topological neighborhood included in FINDCYCLE is large enough then we have $\Delta^{(i+1)}=1$. To estimate the probability for $\Delta^{(i+1)}=1$ we apply the same approach as in the analysis for the case of type (1) and (2) cycles. This yields immediately (observing that we have $\frac{\lambda n}{2}$ cycles instead of $\lambda n$ ):

$$
\operatorname{Pr}\left[\Delta^{(i+1)}=1\right] \geq \frac{1}{2 \ell} \cdot \frac{\ell}{2 n} \cdot \frac{1}{5} \cdot \frac{\lambda n}{2}=\frac{\lambda}{40} .
$$

Our next goal is to show that there are at least $\frac{\lambda s}{80}$ cycles that are half-contained in $S^{(s)}$.

\section{Claim 4.3}

$$
\operatorname{Pr}\left[\sum_{C \in \mathfrak{C}_{3} \cup \mathfrak{C}_{4}} X_{C}^{(s)} \leq \frac{\lambda s}{80}\right] \leq e^{-\frac{\lambda s}{300}}
$$

Proof :

$$
\operatorname{Pr}\left[\sum_{C \in \mathfrak{C}_{4} \cup \mathfrak{C}_{4}} X_{C}^{(s)} \leq \frac{\lambda s}{80}\right] \leq \operatorname{Pr}\left[\sum_{1 \leq i \leq s} \Delta^{(i)} \leq \frac{\lambda s}{80}\right] \leq \operatorname{Pr}\left[\sum_{1 \leq i \leq s} B^{(i)} \leq \frac{\lambda s}{80}\right],
$$

where $\mathrm{B}^{(i)}$ are independent $0-1$ variables with $\operatorname{Pr}\left[\mathrm{B}^{(i)}=1\right]=\frac{\lambda}{40}$. The latter inequality follows from Claim 4.2. We now apply a Chernoff bound [13, inequality (7)] to obtain

$$
\operatorname{Pr}\left[\sum_{C \in \mathfrak{C}_{4} \cup \mathfrak{C}_{4}} X_{C}^{(s)} \leq \frac{\lambda s}{80}\right] \leq \operatorname{Pr}\left[\sum_{1 \leq i \leq s} B^{(i)} \leq\left(1-\frac{1}{2}\right) \cdot \frac{\lambda s}{40}\right] \leq e^{-\frac{\lambda s}{320}} .
$$

Let $W^{(i+1)}$ denote the indicator random variable for the event that there exists $C \in \mathfrak{C}_{3} \cup \mathfrak{C}_{4}$ with $X_{C}^{(i)}=1$ and $Y_{C}^{(i)}=0$ and $Y_{C}^{(i+1)}=1$.

Claim 4.4 Let the outcome of the random choices in round 1 to $i$ of the procedure FINDCYCLE be fixed. If

$$
\sum_{C \in \mathfrak{C}_{3} \cup \mathfrak{C}_{4}} X_{C}^{(i)}>\frac{\lambda s}{80}
$$

then

$$
\operatorname{Pr}\left[W^{(i+1)}\right] \geq \frac{\lambda s}{1600 n}
$$


Proof : We assume that there are more than $\frac{\lambda s}{80}$ cycles that are half-contained in $S^{(i)}$. Again, we use essentially the same approach as in the case of type (1) and (2) cycles. We observe that there is a problem with cycles of type (3). Since the length of the shorter path is 0 there is no directed edge in this path. Thus we have to slightly modify our approach. We use the following sampling scheme for the analysis: Instead of taking the whole topological $2^{j}$-neighborhood of $p^{(i)}$ we choose a number $k$ between 1 and 6 uniformly distributed. If $k$ is between 1 and five we include the $2^{j}$ predecessors and successors of the kth edge incident to $p^{(i)}$. In the case $k=6$ we only include the vertex $p^{(i)}$ in the sample. Then we get that the probability that a cycle $C$ is contained in the sample is at least $\frac{1}{2 \ell} \cdot \frac{\ell}{2 n} \cdot \frac{1}{6}=\frac{1}{24 n}$. We have more than $\frac{\lambda s}{80}$ cycles that are half-contained in $S^{(i)}$. Therefore we obtain that:

$$
\operatorname{Pr}\left[W^{(i+1)}\right] \geq \frac{\lambda s}{1920 \cdot n}
$$

Lemma 4.5 Let $\mathrm{G}$ be a geometric graph for $\mathrm{P}$ with maximum degree five that has at least $\frac{\mathrm{\epsilon}}{200}$ topological $\epsilon$-short cycles of type (3) or (4). Then FINDCYCLE is an algorithm with (expected) query complexity $\mathcal{O}(\sqrt{\mathrm{n} / \epsilon} \log (\mathrm{n} / \epsilon))$ that samples a set $\mathrm{S} \subseteq \mathrm{P},|\mathrm{S}| \geq 1700 \sqrt{\mathrm{n} / \epsilon}+\frac{192000}{\epsilon}$, such that the EMST-completion of the subgraph $\mathrm{G}_{\mathrm{S}^{(2 s)}}$ induced by $\mathcal{S}^{(2 \mathrm{~s})}$ has an $\epsilon$-short topological cycle.

Proof : Let $\lambda=\frac{\epsilon}{200}$ and let $\mathrm{G}$ be a geometric graph for $\mathrm{P}$ with maximum degree five that has at least $\lambda n$ topological $\epsilon$-short cycles of type (3) or (4). By Claim 4.4 the probability that there is a cycle in the EMST-completion of the subgraph induced by $S^{(2 s)}$ is greater than or equal to

$$
1-\left(\operatorname{Pr}\left[\frac{1}{s} \sum_{C \in \mathfrak{C}_{3} \cup \mathfrak{C}_{4}} X_{C}^{(s)} \leq \frac{\lambda}{80}\right]+\left(1-\frac{\lambda s}{1920 n}\right)^{s}\right) .
$$

Choosing $s \geq 1700 \sqrt{n / \epsilon}+\frac{192000}{\epsilon}$, this bound together with Claim 4.3 for $n \geq 4$ implies that the probability that there is a cycle in the EMST-completion of the subgraph induced by $S^{(2 s)}$ is greater than or equal to $1-\left(e^{-2}+e^{-5}\right) \geq \frac{4}{5}$.

The complexity of our tester in Lemma 4.5 is in expectation. We can obtain a tester with a deterministic bound on the query complexity, if we stop our algorithm when the sample size is some constant larger than its expectation. The small additional error can be charged to the failure probability of the algorithm.

Lemma 4.6 Let $\mathrm{G}$ be a well-shaped geometric graph for $\mathrm{P}$. Then there is a property tester that in time $\mathcal{O}\left(\log ^{2}(n / \epsilon) \cdot \sqrt{n / \epsilon}\right)$ and with query complexity of $\mathcal{O}(\log (n / \epsilon) \cdot \sqrt{n / \epsilon})$ accepts the input $\mathrm{G}$ if $\mathrm{G}$ is an EMST of $\mathrm{P}$ and that rejects the input with probability at least $\frac{2}{3}$ if $\mathrm{G}$ is $\in$-far from EMST.

Proof : Follows from Lemmas 3.74.1 and 4.5

\subsection{Extension to general graphs}

The result from Lemma 4.6 can be extended using property testing algorithms from Sections 3.3 3.5 to obtain the following result.

Theorem 3 There is a property tester for the EMST property with a running time of $\left.\mathcal{O}(\sqrt{\mathrm{n} / \epsilon}) \cdot \log ^{2}(\mathrm{n} / \epsilon)\right)$ and with a query complexity of $\mathcal{O}(\sqrt{n / \epsilon} \cdot \log (n / \epsilon))$. 


\section{Conclusions}

There are a few open problems left after our work. We believe that our property testing algorithms have asymptotically optimal or almost optimal complexity, but we leave as an open question whether this is indeed the case. Our algorithms have been using many properties of minimal spanning trees for sets of points on the plane; can one extend our approach to obtain an $\mathrm{o}(\mathrm{n})$-time property testing for EMST in higher dimensions, say, even in $\mathbb{R}^{3}$ ?

\section{References}

[1] J. L. Bentley and T. A. Ottmann. Algorithms for reporting and counting geometric intersections. IEEE Transactions on Computers, C-28:643-647, 1979.

[2] B. Chazelle. A minimum spanning tree algorithm with inverse-Ackermann type complexity. Journal of the ACM, 47(6): 1012-1027, November 2000.

[3] B. Chazelle, R. Rubinfeld, and L. Trevisan. Approximating the minimum spanning tree weight in sublinear time. SIAM Journal on Computing, 34(6): 1370-1379, 2005.

[4] A. Czumaj, F. Ergün, L. Fortnow, A. Magen, I. Newman, R. Rubinfeld, and C. Sohler. Sublinear-time approximation of Euclidean minimum spanning tree. SIAM Journal on Computing, 35(1): 91-109, 2005.

[5] A. Czumaj and C. Sohler. Estimating the weight of metric minimum spanning trees in sublinear-time. In Proceedings of the 36th Annual ACM Symposium on Theory of Computing (STOC), pages 175-183, 2004.

[6] A. Czumaj, C. Sohler, and M. Ziegler. Property testing in computational geometry. In Proceedings of the 8th Annual European Symposium on Algorithms (ESA), pp. 155-166, 2000.

[7] B. Dixon, M. Rauch, and R. E. Tarjan. Verification and sensitivity analysis of minimum spanning trees in linear time. SIAM Journal on Computing, 21(6):1184-1192, 1992.

[8] D. Eppstein. Spanning trees and spanners. In Handbook of Computational Geometry, pp. 425-461. Elsevier Science B.V., 1997.

[9] E. Fischer. The art of uniformed decisions. A primer to property testing. Bulletin of the European Association for Theoretical Computer Science, 75:97 - 126, 2001.

[10] O. Goldreich. Combinatorial property testing (a survey). In P. Pardalos, S. Rajasekaran, and J. Rolim, editors, Proceedings of the DIMACS Workshop on Randomization Methods in Algorithm Design, volume 43 of DIMACS, Series in Discrete Mathematics and Theoretical Computer Science, pages 45-59, 1997. American Mathematical Society, Providence, RI, 1999.

[11] O. Goldreich, S. Goldwasser, and D. Ron. Property testing and its connection to learning and approximation. Journal of the ACM, 45(4):653-750, 1998.

[12] O. Goldreich and D. Ron. Property testing in bounded degree graphs. Algorithmica, 32(2): 302-343, 2002. 
[13] T. Hagerub and C. Rüb. A guided tour to Chernoff bounds. Information Processing Letters, 33:305308, 1989.

[14] D. R. Karger, P. N. Klein, and R. E. Tarjan. A randomized linear-time algorithm to find minimum spanning trees. Journal of the ACM, 42(2): 321-328, March 1995.

[15] V. King. A simpler minimum spanning tree verification algorithm. Algorithmica, 18:263-270, 1997.

[16] D. G. Kirkpatrick and J. D. Radke. A framework for computational morphology. In Computational Geometry, pages 217-248, North-Holland, 1985.

[17] J. Komlós. Linear verification for spanning trees. Combinatorica, 5(1): 57-65, 1985.

[18] M. Parnas and D. Ron. Testing the diameter of graphs. Random Structures and Algorithms, 20(2): 165-183, March 2002.

[19] S. Pettie and V. Ramachandran. An optimal minimum spanning tree algorithm. Journal of the ACM, 49(1): 16-34, 2002.

[20] D. Ron. Property testing. In Handbook of Randomized Algorithms, volume II, pages 597-649, Kluwer Academic Publishers, 2001.

[21] R. Rubinfeld and M. Sudan. Robust characterization of polynomials with applications to program testing. SIAM Journal on Computing, 25(2):252-271, 1996.

[22] M. I. Shamos and D. Hoey. Closest-point problems. In Proceedings of the 16th Annual IEEE Symposium on Foundations of Computer Science (FOCS), pages 151-162, 1975.

[23] R. E. Tarjan. Data structures and network algorithms. In CBMS-NSF Regional Conference Series in Applied Mathematics, volume 44. Society for Industrial and Applied Mathematics (SIAM), 1983. 